\title{
A auto-eficácia como mediadora da melhora em índices clínicos de saúde oral
}

\section{Self-efficacy as a mediator of improvement in oral health clinical indices}

\author{
Gislaine Afonso de SOUZA* \\ Angela Maria MONTEIRO DA SILVA** \\ Rogério GALVÃO***
}

\begin{abstract}
SOUZA, G. A. de; MONTEIRO DA SILVA, A. M.; GALVÃO, R. A auto-eficácia como mediadora da melhora em índices clínicos de saúde oral. Pesqui Odontol Bras, v. 16, n. 1, p. 57-62, jan./mar. 2002.

O conceito de auto-eficácia (AE) foi introduzido em 1977 por BANDURA ${ }^{2}$. Com ampla utilização na área de Saúde, ele tem se revelado um poderoso preditor em vários domínios de comportamentos, tais como: parar de fumar, perder peso, participar de programas de prevenção, entre outros. Este estudo investigou se uma intervenção psicológica produz melhora de indices clínicos em pacientes prestes a receberem tratamento odontológico. Previu-se que o grupo de teste (GT) melhoraria mais do que o grupo de controle (GC) nos índices clínicos de placa e sangramento e que a AE mediaria esta melhora. Os 30 participantes do GT, além do procedimento odontológico padrão, receberam uma intervenção psicológica baseada no modelo de PROCHASKA; DiCLEMENTE ${ }^{12}$ (1983). Os 30 participantes do GC receberam somente o procedimento odontológico padrão. Os resultados indicaram que os dois grupos apresentaram uma redução significativa dos índices clínicos do pré- para o pós-teste. Porém, os escores 2 e 3 de placa e sangramento sofreram um decréscimo significativamente maior no GT comparado ao GC $(U=197,00, p=0,0001$ e $U=179,00, p=0,0001$, respectivamente). Apenas o GT apresentou um incremento significativo na AE de pré- para pós-tratamento $(Z=-3,58$, $\mathrm{p}<0,0001)$. No entanto, não houve diferença entre os grupos em termos de aumento de AE do pré- para o pós-teste. Concluindo, os resultados revelaram que a intervenção psicológica foi efetiva e sugerem a possibilidade de que outros fatores além da AE medeiem a melhora significativa do GT em índices clínicos.
\end{abstract}

UNITERMOS: Auto-eficácia; Índice CPO; Odontopatias; Saúde bucal; Psicologia.

\section{INTRODUÇÃO}

As pessoas são capazes de exercer, ao menos em parte, um controle sobre a sua saúde. Por exemplo, parar de fumar e passar a manter uma higiene oral de elevado padrão são mudanças comportamentais que favorecem a saúde oral.

$\mathrm{O}$ conhecimento a respeito da maneira como as pessoas organizam seus pensamentos e ações referentes a sua saúde é fundamental para conduzi-las a uma prevenção efetiva de doenças e transtornos. Crenças, atitudes e outras variáveis cognitivas são freqüentemente consideradas os maiores determinantes da auto-regulação de comportamento. Dentre os modelos da Psicologia que têm enfatizado o papel da cognição (por exemplo, pensamentos e conceitos do indivíduo) na regulação do comportamento, destaca-se o modelo da auto-eficácia ${ }^{21}$.
O conceito da auto-eficácia (AE) tem gerado muito interesse e alguma controvérsia desde que foi introduzido por BANDURA ${ }^{2}$, em 1977, e tem se mostrado uma variável chave na Psicologia clínica, educacional e social, do desenvolvimento e da saú$\mathrm{de}^{15}$. A AE percebida foi inicialmente definida como a crença do indivíduo de que ele pode executar um comportamento específico ou tarefa futura ${ }^{2}$. Mais recentemente, BANDURA ${ }^{3}$ (1997) declara que a AE se refere às crenças do indivíduo acerca de suas capacidades para organizar e executar os cursos de ação necessários para produzir determinados resultados.

Na promoção da mudança comportamental ligada à saúde, é preciso fornecer às pessoas não só as razões para mudar o comportamento de saúde mas também os meios e recursos para fazê-lo. A auto-regulação efetiva do comportamento requer

\footnotetext{
${ }^{*}$ Doutoranda em Saúde Coletiva pela Universidade do Estado do Rio de Janeiro.

** Professora Doutora do Mestrado em Psicologia; ***Professor Adjunto do Departamento de Odontologia - Universidade Gama Filho.
} 
SOUZA, G. A. de; MONTEIRO DA SILVA, A. M.; GALVÃO, R. A auto-eficácia como mediadora da melhora em índices clínicos de saúde oral. Pesqui Odontol Bras, v. 16, n. 1, p. 57-62, jan./mar. 2002.

certas habilidades de automotivação e autodireção. Além do mais, há uma diferença entre possuir certas habilidades e ser capaz de usá-las efetiva e consistentemente sob condições adversas. O sucesso requer, portanto, não apenas habilidades mas também uma forte crença na própria capacidade de exercer controle. A teoria da AE abarca todos esses aspectos da mudança comportamental favorável à saúde.

Vários estudos indicam que a $\mathrm{AE}$ tem se mostrado um poderoso preditor em vários domínios da saúde, tais como: parar de fumar, perder peso, controlar a dor, recuperar-se de infarto e participar de diversos programas de prevenção de doença $^{11,15}$.

Há, na literatura, poucos estudos sobre a AE na área de Saúde Oral. Tais estudos geralmente visam prevenir ou controlar as doenças dentais mais comuns: a cárie e a doença periodontal. REISINE; LITT $^{13}$ (1993), por exemplo, investigaram quatro conceitos teóricos que possivelmente ajudariam a ampliar os conhecimentos sobre o comportamento de higiene oral e o risco de cárie: classe social, eventos estressantes de vida, AE e locus de controle. Os resultados indicaram que pais com locus de controle externo elevado, AE mais baixa, baixa renda e altos niveis de estresse tinham filhos com maior risco de cárie. Os autores recomendam aos dentistas encorajar seus pacientes a desenvolverem o locus de controle interno (a pessoa com locus de controle interno acredita ser responsável pelo que lhe acontece e que há coisas que ela pode fazer para melhorar a sua saúde dental).

A utilização da teoria da $\mathrm{AE}$ somada à teoria da ação racional para predizer o comportamento de saúde oral foram testadas por TEDESCO et al. ${ }^{19}$ (1993). Os resultados indicaram que as medidas cognitivo-sociais (variáveis da teoria da ação racional e AE) foram capazes de predizer até, no máximo, $11 \%$ da variância dos índices de placa e sangramento gengival e, no máximo, $51 \%$ do comportamento de saúde oral auto-relatado. A AE, quando acrescentada às variáveis da teoria da ação racional, explicou uma variância adicional significativa do comportamento de saúde oral auto-relatado, mas não acrescentou nada em termos da variância de acúmulo de placa e sangramento gengival.

STEWART et al. ${ }^{17}$ (1996) compararam e avaliaram a efetividade de duas intervenções planejadas para mudar a $\mathrm{AE}$ de escovação e o uso de fio dental. Havia três grupos, um de controle (tratamento dentário e treinamento em higiene oral), um grupo que recebeu uma intervenção educativa (tratamento dentário, e informações sobre a doença periodontal) e outro que foi submetido a uma intervenção psicossocial. Este grupo, além de tratamento dentário e treinamento em higiene oral, recebeu quatro sessões com um psicólogo baseadas no aconselhamento motivacional de MILLER $^{10}$ (1983) e no modelo de estágios de mudança de PROCHASKA; DiCLEMENTE ${ }^{12}$ (1983). Os resultados demonstraram que não houve mudanças significativas no conhecimento de saúde bucal no grupo de controle. Houve mudanças estatisticamente significativas e similares quanto ao aumento de conhecimento de saúde bucal, tanto para o grupo que recebeu orientação educativa, quanto para o grupo que passou por uma intervenção psicológica. Os três grupos apresentaram um aumento da $\mathrm{AE}$ de uso de fio dental do pré- para o pós-teste. A magnitude das mudanças em AE foi similar quando o grupo de controle foi comparado com o grupo da intervenção educativa. Em contrapartida, o grupo de intervenção psicossocial demonstrou um aumento significativamente maior na $\mathrm{AE}$ de uso de fio dental, quando comparado com o grupo da intervenção educativa. Esses dados sugerem que o aumento da $\mathrm{AE}$ pode ser um importante componente de intervenções destinadas a mudar comportamentos de saúde oral, e que a $\mathrm{AE}$ pode servir para avaliar e comparar a eficácia dessas intervenções.

Devido à maior incidência de periodontite severa em pacientes com diabetes mellitus insulino-dependentes, visitas regulares ao dentista e cuidados diários com a higiene oral são de suma importância para tais pacientes. SYRJÄLÄ et al. ${ }^{18}$ (1999) desenvolveram um método para avaliar a AE dental, para estudar a sua relação com o comportamento de saúde oral auto-relatado e com o acúmulo de placa dental. A relação entre o comportamento de saúde oral e níveis de hemoglobina glicosilada (HbA1c) também foi investigada. Os resultados mostraram que a $\mathrm{AE}$ de escovação, a $\mathrm{AE}$ de limpeza interproximal e a AE de visita odontológica se relacionaram significativamente com o comportamento de saúde oral auto-relatado (escovação e uso de fio dental). O índice de placa visivel correlacionou-se inversamente com a $\mathrm{AE}$ de escovação e com a $\mathrm{AE}$ de visita dental. A AE de limpeza interproximal correlacionou-se inversamente com a idade. Pacientes com alta freqüência de escovação e baixo índice de placa visivel apresentavam 
SOUZA, G. A. de; MONTEIRO DA SILVA, A. M.; GALVÃO, R. A auto-eficácia como mediadora da melhora em índices clínicos de saúde oral. Pesqui Odontol Bras, v. 16, n. 1, p. 57-62, jan./mar. 2002.

menor nivel de HbA1c. Por fim, niveis mais altos de $\mathrm{AE}$ de visita dental estavam relacionados a melhores niveis educacionais e profissionais. Concluiu-se que a $\mathrm{AE}$ percebida do comportamento de saúde oral está associada aos níveis clínicos de higiene oral. Associações entre baixo controle metabólico dos diabéticos (altos níveis de $\mathrm{HbA1c}$ ), baixa freqüência de escovação e altos níveis de placa dental sugerem que a educação dental, incluindo o aumento da $\mathrm{AE}$, é de grande importância, especialmente para o portador de diabetes mellitus. Os autores ressaltaram, ainda, que os cirurgiões-dentistas podem promover o aumento da $\mathrm{AE}$ dental empregando princípios e técnicas psicológicas.

Muitas vezes, sem fazer uso explícito de modelos teóricos da Psicologia, os dentistas têm desenvolvido programas para a promoção de saúde oral. Tais programas envolvem o emprego do modelo educacional que vem sendo freqüentemente adotado pelos dentistas há mais de trinta anos (ensinamentos verbais, demonstrativos de técnicas de higiene oral e instruções sobre a etiologia das doenças dentais). Entretanto, esses programas baseados no modelo educacional parecem não produzir mudanças positivas no comportamento da higiene oral por muito tempo ${ }^{1,7}$. Sabendo que as doenças bucais (por exemplo, cárie e doença periodontal), geralmente assintomáticas no início, podem ser prevenidas pelo uso correto das técnicas de higiene oral e manutenção de um alto nivel de higiene oral, a classe odontológica pode lançar mão de outros recursos para atingir os seus objetivos. Nesse processo, o conhecimento psicológico, especialmente a teoria da $\mathrm{AE}$, pode ser de grande valia.

$O$ presente estudo visou investigar se uma intervenção psicossocial, possivelmente tendo a $\mathrm{AE}$ como mediadora, conduz a mudanças positivas em termos de redução de índices clínicos em pacientes prestes a receberem tratamento odontológico.

\section{MATERIAL E MÉTODO}

O estudo foi realizado em uma amostra total de 60 pacientes da Clínica Odontológica da Universidade Gama Filho (UGF), portadores de cárie e/ou doença periodontal (presença de, ao menos, um sítio com profundidade de bolsa $\geq 5 \mathrm{~mm}$ em pelo menos 3 quadrantes), prestes a receberem o tratamento odontológico indicado. Participaram 30 homens e 30 mulheres na faixa etária entre 18 e 61 anos. Foram excluídos indivíduos que não apresentavam no mínimo 12 dentes naturais, com dis- túrbios psicóticos ou problemas motores que dificultassem a escovação e o uso de fio dental. Todos os participantes estavam havia pelo menos 6 meses sem tratamento odontológico. Foram também excluídos pacientes que fizeram uso de antibióticos nos últimos 6 meses, com doenças sistêmicas relacionadas com o processo saúde-doença periodontal como, diabete, epilepsia e hipertensão, e pacientes gestantes. Pacientes com menos de $20 \%$ de placa visivel nas faces de seus dentes não participaram do estudo. Todos os participantes possuiam grau de instrução variando de ensino fundamental completo a superior completo.

Cada participante teve os seus índices de placa $^{16}$ e gengival ${ }^{8}$, respectivamente, medidos por um examinador. Em seguida, o participante respondeu ao questionário de $\mathrm{AE}$ ligeiramente modificado de higiene oral ${ }^{9}$ e ao questionário sócio-demográfico. $\mathrm{O}$ instrumento para a medida da $\mathrm{AE}$ foi previamente adaptado pelos autores do presente estudo, tendo apresentado elevada precisão de consistência interna ( $\alpha$ de Crombach igual a 0,98). Os participantes foram randomicamente divididos em dois grupos: grupo de controle (GC) e grupo de teste (GT).

No início do estudo, os participantes de ambos os grupos receberam um treino de escovação e uso de fio dental.

O GC recebeu o procedimento padrão de um dentista (orientação sobre as causas e conseqüências da doença periodontal e da cárie, dieta, instrução de higiene oral e tratamento dentário).

O GT, além de receber o procedimento padrão de um dentista, foi o grupo que recebeu a intervenção psicológica. Foram 4 sessões semanais de aproximadamente 20 minutos de duração, em que foram utilizadas técnicas cognitivo-comportamentais: a) auto-monitoramento (por exemplo, o paciente aprende a identificar placa, dar atenção às áreas de dificil acesso, observar sangramento durante a higiene oral); b) manejo de contingências (recompensar os comportamentos favoráveis à saúde); c) controle de estímulos (usar dicas para se lembrar de executar as operações de higiene oral); d) plano de metas (estabelecer objetivos a serem gradualmente alcançados) e e) reforço (por exemplo, o dentista deve estar sempre dando "feedback" ao paciente, recompensando e valorizando cada objetivo alcançado).

Todos os participantes do estudo foram submetidos ao pós-teste após 3 meses de tratamento. Responderam novamente aos mesmos questioná- 
SOUZA, G. A. de; MONTEIRO DA SILVA, A. M.; GALVÃO, R. A auto-eficácia como mediadora da melhora em índices clínicos de saúde oral. Pesqui Odontol Bras, v. 16, n. 1, p. 57-62, jan./mar. 2002.

rios do pré-teste e foram reexaminados clinicamente quanto aos índices de placa e gengival.

\section{Análise dos dados}

A análise dos dados foi feita usando-se o pacote estatístico comercial SPSS. Foram realizados testes de Mann-Whitney para comparar os dois grupos quanto à $\mathrm{AE}$, placa bacteriana e sangramento gengival. Produziu-se testes de Wilcoxon para comparar os dados de pré- e pós-teste de ambos os grupos quanto à $\mathrm{AE}$, placa e ao sangramento. Para todas as análises, usou-se um nivel de significância igual a $5 \%$.

\section{RESULTADOS}

Os dados básicos das variáveis do estudo são apresentados na Tabela 1 .

Foram feitas comparações dos níveis de AE e dos índices clínicos entre os grupos (de teste e de controle) e de pré- para pós-teste em ambos os grupos.

Uma análise de Mann-Whitney indicou que o GT não teve um incremento significativamente maior na $\mathrm{AE}$ do pré- para o pós-teste quando comparado com o GC $(\mathrm{U}=353,50, \mathrm{p}=0,15)$. No entanto, o GT apresentou um decréscimo significativamente maior do que o GC nos escores 2 e 3 de placa e de sangramento do pré- para o pós-teste: $\mathrm{U}=197,00, \mathrm{p}<0,0001$ e $\mathrm{U}=179,50, \mathrm{p}<0,0001$, respectivamente.

TABELA 1 - Niveis de auto-eficácia (AE), níveis de placa e sangramento no pré- e no pós-teste para ambos os grupos.

\begin{tabular}{l|c|c|c|c}
\hline \hline \multicolumn{1}{c|}{ Variável } & Grupo & $\mathrm{n}$ & Média & $\mathrm{dp}$ \\
\hline $\begin{array}{l}\text { Escore total de AE } \\
\text { do pré-teste }\end{array}$ & Teste & 30 & 74,33 & 17,72 \\
\cline { 2 - 5 } & Controle & 30 & 74,53 & 18,91 \\
\hline $\begin{array}{l}\text { Escore total de AE } \\
\text { do pós-teste }\end{array}$ & Teste & 30 & 85,50 & 14,32 \\
\cline { 2 - 5 } & Controle & 30 & 81,07 & 16,69 \\
\hline \multirow{2}{*}{$\begin{array}{l}\text { Nível de placa no } \\
\text { pré-teste }\end{array}$} & Teste & 30 & 45,17 & 17,69 \\
\cline { 2 - 5 } & Controle & 30 & 39,58 & 21,06 \\
\hline \multirow{2}{*}{$\begin{array}{l}\text { Nível de placa no } \\
\text { pós-teste }\end{array}$} & Teste & 30 & 16,53 & 12,01 \\
\cline { 2 - 5 } & Controle & 30 & 27,83 & 15,95 \\
\hline $\begin{array}{l}\text { Nível de sangra- } \\
\text { mento no pré-teste }\end{array}$ & Teste & 30 & 45,15 & 16,26 \\
\cline { 2 - 5 } & Controle & 30 & 40,63 & 18,91 \\
\hline $\begin{array}{l}\text { Nível de sangra- } \\
\text { mento no pós-teste }\end{array}$ & Teste & 30 & 10,66 & 6,87 \\
\cline { 2 - 5 } & Controle & 30 & 18,91 & 12,66 \\
\hline \hline
\end{tabular}

O teste de Wilcoxon para os dados pareados indicou que a $\mathrm{AE}$ de pós-teste foi significativamente maior do que a AE de pré-teste no GT: $Z=-3,58$, $p<0,0001$. Os escores de placa 2 e 3 apresentaram-se significativamente reduzidos do pré- para o pós-teste $(Z=-4,78, p<0,0001)$ no GT. Os escores de sangramento deste grupo também sofreram redução significativa de pré- para o pós-teste $(\mathrm{Z}=-4,78, \mathrm{p}<0,0001)$.

No GC, a AE de pós-teste, quando comparada a de pré-teste, não aumentou significativamente $(Z=-1,76, p=0,08)$. Todavia, os escores 2 e 3 de placa e sangramento sofreram uma diminuição significativa: $Z=-3,40, p<0,001$ e $Z=-4,78$, $\mathrm{p}<0,0001$, respectivamente.

\section{DISCUSSÃO E CONCLUSÕES}

Os dados do presente estudo indicam que os dois grupos apresentaram um decréscimo significativo dos níveis de placa e de sangramento do prépara o pós-teste. Porém, o GT apresentou uma melhora significativamente maior do que o GC nos dois índices clínicos. Estes achados indicam que a intervenção psicológica é um tratamento coadjuvante efetivo, produzindo uma melhora significativa quando combinada com o tratamento odontológico. No entanto, a hipótese de que a $\mathrm{AE}$ medeia a redução dos índices clínicos de pré- para pós-tratamento não foi totalmente confirmada. Embora tenha havido um incremento significativo de AE do pré- para o pós-teste apenas no GT, não há diferença significativa entre os dois grupos quanto ao aumento da AE de pré- para pós-teste. Tais achados sugerem que outros fatores além da $\mathrm{AE}$ possivelmente sejam responsáveis pela melhora significativamente maior dos índices clínicos no GT, em comparação com o GC. Por outro lado, embora a diferença de $\mathrm{AE}$ de pós- para pré-teste (incremento de $\mathrm{AE}$ ) não tenha variado entre os grupos, o aumento significativo de AE de pré- para pós-teste no GT pode ter sido, ao menos em parte, responsável pela melhora maior deste grupo nos índices clínicos quando comparado com o GC. O presente estudo difere do conduzido por STEWART et al. ${ }^{17}$ (1996) por ter incluído em sua metodologia a coleta de indices de placa ${ }^{16}$ e sangramento gengival ${ }^{8}$. Esta inclusão permitiu examinar a associação da intervenção psicológica com níveis de $\mathrm{AE}$ e índices clínicos. 
SOUZA, G. A. de; MONTEIRO DA SILVA, A. M.; GALVÃO, R. A auto-eficácia como mediadora da melhora em índices clínicos de saúde oral. Pesqui Odontol Bras, v. 16, n. 1, p. 57-62, jan./mar. 2002.

A intervenção psicológica foi baseada no modelo de estágios de mudança de PROCHASKA; DiCLEMENTE $^{12}$ (1983). Segundo este modelo, quatro princípios são fundamentais para o processo de mudança: a) responsabilidade individual (reconhecer o problema e que algo precisa ser feito); b) atribuição interna (responsabilizar-se pelo seu status atual de saúde oral, que depende de ir regularmente ao dentista, atingir e manter elevado padrão de higiene oral, dentre outros); c) decisão pessoal (considerar-se responsável e capaz de tomar decisões a respeito de um curso de ação para melhorar a saúde oral) e d) criar dissonância cognitiva (ajudar no reconhecimento de inconsistências ou incongruências entre comportamentos, crenças, atitudes e sentimentos). Por exemplo, um indivíduo que acredita que a escovação é importante para a sua saúde oral, mas não a executa devidamente, está experimentando desacordo ou dissonância cognitiva.

Como a intervenção psicológica visou alcançar as metas ligadas a esses principios, fatores tais como o aumento da responsabilidade individual, da atribuição interna e o reconhecimento de dissonância cognitiva podem ter sido, em parte, responsáveis pelo sucesso da intervenção. Assim, futuros estudos poderiam avaliar a influência desses fatores na redução dos índices clínicos. Tais estudos envolveriam: a) a medida de $\mathrm{AE}$ e das outras supramencionadas variáveis passiveis de explicar a mudança de índices clínicos; b) o emprego de modelos hierárquicos de análise. Além do mais, sugere-se estudos prospectivos para verificar se os pacientes que recebem a intervenção psicológica mantêm os seus novos hábitos, de modo que a saúde oral seja mantida por um longo período.

Outro fator que talvez possa ter contribuído para o sucesso da intervenção diz respeito ao aumento da orientação direta e individual de cada participante do GT em comparação com o GC. Por exemplo, COSTA et al. ${ }^{5}$ (1999), SABA-CHUJFI et $a l .{ }^{14}$ (1992) e COUTO et al. ${ }^{6}$ (1992) enfatizaram a relevância do contato direto com o paciente para o controle de placa bacteriana na prevenção da cárie e da doença periodontal.

A melhora dos índices clínicos de ambos os grupos está acima da média descrita na literatura ${ }^{4}$. Os trabalhos sobre educação e promoção em saúde bucal apresentam uma média de melhora no índice de placa, após a implementação de programas de promoção de saúde bucal, de aproximadamente $17,8 \%$. VASCONCELLOS ${ }^{20}$ (1999), em um programa familiar de promoção de saúde bucal, obteve uma redução do índice de placa do pré- para o pós-teste, no grupo experimental, de $25 \%$. No presente estudo observou-se uma redução do índice de placa no GT de $63,4 \%$ e de $29,7 \%$ no GC. Resultados semelhantes foram obtidos com o índice de sangramento gengival (GT $=76,4 \%$ e GC $=53,5 \%$ ). Este índice é de grande importância clínica, pois demonstra o verdadeiro hábito do paciente em realizar o seu controle de placa.

A situação de saúde ou doença é melhor avaliada pelo indice de sangramento porque o índice de placa tem a desvantagem, ao ser avaliado sozinho, de mostrar apenas a situação no momento do exame, podendo não refletir o comportamento de higiene oral usual do paciente.

Sob o ponto de vista clínico e da literatura revista, parece que a intervenção psicológica no presente estudo contribuiu muito para a redução dos índices clínicos. Presume-se que os pacientes do GT foram os mais motivados e os que aprenderam a melhor se auto-avaliar. Isto possivelmente gerou melhora da autocrítica, maior conhecimento de si próprio e maior responsabilidade sobre os comportamentos favoráveis à saúde oral.

Em suma, a presença da placa bacteriana constitui-se no agente etiológico determinante da cárie e da doença periodontal. Sendo assim, meios que visam transmitir hábitos de higiene oral para $\mathrm{o}$ controle da placa são de grande valia. É necessário que as instituições formadoras de cirurgiões-dentistas, bem como os programas de promoção de saúde, formem profissionais conscientes de sua importância na promoção da mudança de comportamento favorável à saúde oral do paciente. O presente estudo também indica que teorias psicológicas, tais como o modelo de estágios de Prochaska e DiClemente e a teoria da $\mathrm{AE}$, podem ser úteis. Finalmente, pode-se concluir que um profissional envolvido com questões psicológicas, sociais e culturais terá melhores condições para transformar hábitos antigos e promover hábitos mais saudáveis e duradouros. 
SOUZA, G. A. de; MONTEIRO DA SILVA, A. M.; GALVÃO, R. A auto-eficácia como mediadora da melhora em índices clínicos de saúde oral. Pesqui Odontol Bras, v. 16, n. 1, p. 57-62, jan./mar. 2002.

SOUZA, G. A. de; MONTEIRO DA SILVA, A. M.; GALVÃO, R. Self-efficacy as a mediator of improvement in oral health clinical indices. Pesqui Odontol Bras, v. 16, n. 1, p. 57-62, jan./mar. 2002.

The concept of self-efficacy (SE) was developed by BANDURA ${ }^{2}$, in 1977. SE has been widely utilized in health care and has shown to be a powerful predictor in various domains of behavior such as in smoking cessation, weight control and participation in programs of disease prevention. This study investigated if a psychosocial intervention fosters improvements in clinical indices in patients who are about to receive dental treatment. It was predicted that the experimental group (EG) would show a greater progress as to plaque and bleeding clinical indices than the control group (CG), and SE would mediate that improvement. Besides the standardized dental procedures, the 30 participants from the EG received a psychosocial intervention based on the PROCHASKA; DiCLEMENTE's ${ }^{12}$ model (1983). The 30 participants in the CG received only standardized dental procedures. The results revealed that the prevalence of scores 2 and 3 of the plaque and bleeding indices underwent a significant decrease in the $E G$, in relation to the $C G(U=97.00, p=0.0001$ and $\mathrm{U}=179.00, \mathrm{p}=0.0001$, respectively). Only the subjects from the EG presented a relevant increase of SE, considering the pre- and post-treatment periods $(Z=-3.58, \mathrm{p}<0.0001)$. Yet, there was no difference between both groups as to the increase of SE between the pre- and post-testing periods. In short, the results showed that psychosocial intervention was effective and suggest that other factors besides SE may mediate a relevant improvement of clinical indices in the EG.

UNITERMS: Self-efficacy; DMF index; Tooth diseases; Oral health; Psychology.

\section{REFERÊNCIAS BIBLIOGRÁFICAS}

1. ANERUD, A. The short- and long-term effect of A-V motivation, motivation by dentist, and motivation by dental hygienist. J Periodontal Res, v. 4, n. 2, p. 171, Mar. 1969.

2. BANDURA, A. Self-efficacy: towards a unifying theory of behavioral change. Psychol Rev, v. 84, n. 2, p. 191-215, Mar. 1977.

3. BANDURA, A. Self-efficacy: the exercise of control. New York: W. H. Freeman and Company, 1997.

4. BROWN, L. F. Research in dental health education and health promotion: a review of literature. Health Educ, v. 21, n. 1, p. 83-102, Spring 1992.

5. CostA, I. C. C.; UNFER, B.; OliveiRA, A. G. R. C. et al. Investindo na promoção da saúde: programas de saúde (PSF) e agentes comunitários (PACS), uma análise de performance em oito municipios brasileiros. Revista da ABOPREV, v. 1, n. 1, p. 30-37, Nov. 1998.

6. COUTO, J. L.; COUTO, R. S.; DUARTE, C. A. Motivação do paciente: avaliação dos recursos didáticos da cárie e doença periodontal. RGO (Porto Alegre), v. 40, n. 2, p. 143-150, mar./abr. 1992.

7. GJERMO, P. Audio-visual motivation and oral hygiene instruction. The effect upon gingival status and oral cleanliness in 15-year-old children. J Periodontal Res, v. 23, n. 2, p. 253-262, Mar. 1967.

8. LÖE, H.; SILNESS, J. Periodontal disease in pregnancy. I prevalence and severity. Acta Odontol Scand, v. 21, n. 6, p. 533-551, Dec. 1963.

9. MAIBACH, E.; MURPHY, D. A. Self-efficacy in health promotion research and practice: conceptualization and measurement. v. 23, p. 37-49, 1995 apud Community Dent Oral Epidemiol, v. 25, n. 5, p. 338, Oct. 1997.

10. MILlER, W. Motivational interviewing with problem drinkers. Behav Psychother, v. 11, p. 147-172, 1983.

11. O'LEARY, A. Self-efficacy and health. Behav Res Ther, v. 23, n. 4, p. 437-451, Apr. 1985.
12. PROChASKA, J. O.; DiClEMENTE, C. C. Stages and processes of self-change of smoking: toward an integrative model of change. J Consult Clin Psychol, v. 51, n. 3, p. 390-395, June 1983.

13. REISINE, S.; LITT, M. Social and psychological theories and their use for dental practice. Int Dent J, v. 43, n. 3, p. 279-287, June 1993.

14. SABA-CHUJFI, E.; DILVA, E. C. Q.; SABA, M. E. C. Avaliação dos métodos de motivação: educação em higiene bucal. RGO (Porto Alegre), v. 40, n. 2, p. 87-90, mar./abr. 1992.

15. SCHWARZER, R.; FUCHS, R. Self-efficacy and health behaviours. In: CONNER, M. E.; NORMAN, P. Predicting health behaviour. Buckinghand : Open University Press, 1996. p. 163-196.

16. SILNESS, J.; LÖE, H. Periodontal disease in pregnancy. II correlation between oral hygiene and periodontal condition. Acta Odontol Scand, v. 22, n. 1, p. 121-135, Feb. 1964.

17. STEWART, J. E.; WOLFE, G. R.; MAEDER, L. et al. Changes in dental knowledge and self-efficacy scores following interventions to change oral hygiene behavior. Patient Educ Couns, v. 27, n. 3, p. 269-277, Apr. 1996.

18. SYRJÄLÄ, A.-M. H.; KNECKT, M. C.; KUNUUTTILA, M. L. E. Dental self-efficacy as a determinant to oral health behaviour, oral hygiene and $\mathrm{HbA} 1 \mathrm{c}$ level among diabetic patients. J Clin Periodontol, v. 26, n. 9, p. 616-621, Sept. 1999.

19. TEDESCO, L. A.; KEFFER, M. A.; DAVIS, E. L. et al. Self-efficacy and reasoned action: predicting oral health status and behaviour at one-, three-, and six-month intervals. Psychol Health, v. 8, p. 105-121, 1993.

20. VASCONCELLOS, I. C. Programa familiar de promoção de saúde. Rio de Janeiro, 1999. Dissertação (Mestrado) - Faculdade de Psicologia, Universidade Gama Filho.

21. WOLFE, G. R.; STEWART, J. E.; HARTZ, G. W. Relationship of dental coping beliefs and oral hygiene. Community Dent Oral Epidemiol, v. 19, n. 2, p. 112-115, Apr. 1991. 